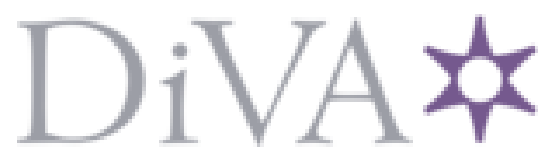

http://www.diva-portal.org

This is the published version of a paper published in Physical Review A. Atomic, Molecular, and Optical Physics.

Citation for the original published paper (version of record):

Niskanen, J., Carravetta, V., Vahtras, O., Ågren, H., Aksela, H. et al. (2010)

Experimental and theoretical study of core-valence double photoionization of OCS.

Physical Review A. Atomic, Molecular, and Optical Physics, 82(4): 043436

http://dx.doi.org/10.1103/PhysRevA.82.043436

Access to the published version may require subscription.

N.B. When citing this work, cite the original published paper.

Permanent link to this version:

http://urn.kb.se/resolve?urn=urn:nbn:se:uu:diva-133719 


\title{
Experimental and theoretical study of core-valence double photoionization of OCS
}

\author{
J. Niskanen,,${ }^{1,2}$ V. Carravetta, ${ }^{3}$ O. Vahtras, ${ }^{2}$ H. Ågren, ${ }^{2}$ H. Aksela, ${ }^{1}$ E. Andersson, ${ }^{4}$ L. Hedin,${ }^{4}$ P. Linusson, ${ }^{5}$ J. H. D. Eland, ${ }^{6,4}$ \\ L. Karlsson, ${ }^{4}$ J.-E. Rubensson, ${ }^{4}$ and R. Feifel ${ }^{4}$ \\ ${ }^{1}$ Department of Physics, University of Oulu, Box 3000, FIN-90014 Oulu, Finland \\ ${ }^{2}$ Department of Theoretical Chemistry, School of Biotechnology, Royal Institute of Technology, SE-10691 Stockholm, Sweden \\ ${ }^{3}$ Institute of Chemical Physical Processes, CNR, via Moruzzi 1, I-56124, Pisa, Italy \\ ${ }^{4}$ Department of Physics and Astronomy, Uppsala University, Box 516, SE-75120 Uppsala, Sweden \\ ${ }^{5}$ Department of Physics, Stockholm University, AlbaNova University Centre, SE-10691 Stockholm, Sweden \\ ${ }^{6}$ Department of Chemistry, Physical and Theoretical Chemistry Laboratory, Oxford University, South Parks Road, \\ Oxford OX1 3QZ, United Kingdom
}

(Received 11 August 2010; published 27 October 2010)

\begin{abstract}
$\mathrm{O} 1 s, \mathrm{C} 1 s$, and S $2 p$ core-valence double ionization electron spectra of the OCS molecule have been obtained experimentally by a time-of-flight photoelectron-photoelectron coincidence spectroscopy technique. In order to analyze and assign the spectral features observed, we present a protocol for computing core-valence ionization energies of such systems. The protocol is based on a restricted active space multiconfigurational self-consistent field (MCSCF) methodology with a freeze-relax procedure to guarantee a correct core-valence state root index without variational collapse. Corrections for extended dynamical correlation and core-core correlation, respectively, are made by multiconfigurational perturbation theory and by uncontracted basis set Møller-Plesset theory. Envisioning applications to larger molecules, a spin-restricted open-shell density functional method is also applied for the lowest core-valence energies. Furthermore, cross sections through a scheme for computing multiatom Auger transitions generating core-valence holes are presented. We find that the procedure outlined is capable of deriving the energy onset of core-valence ionization within a fraction of an $\mathrm{eV}$ and that assignments can be made of the most salient spectral features.
\end{abstract}

DOI: 10.1103/PhysRevA.82.043436

PACS number(s): 33.80.Eh, 33.70.Ca, 34.50.Gb

\section{INTRODUCTION}

Our perception of fundamental molecular processes and new aspects of matter are most often driven by emerging measurement technologies. One example of this assertion is given by double ionization photoelectron spectroscopy (DIPES) that provides information about the energy distribution of dicationic states of atoms and molecules and the probability for emission of two electrons on the absorption of a single incident photon (see, e.g., Refs. [1-6] and references therein). In the strong-field regime, two-photon DIPES can be used in double core-hole spectroscopy, theoretically studied by Cederbaum and coworkers [7-9] and by Ågren and Jensen [10]. DIPES is based on coincidence detection of two (or more) electrons that are created in the same process and can be obtained in an efficient way by the time-of-flight photoelectron-photoelectron coincidence (TOF-PEPECO) spectroscopy method recently introduced by Eland et al. [1]. This technique makes use of a highly efficient magnetic-bottle electron spectrometer [11] to reveal signals from weak processes, like, e.g., singlephoton core-valence double photoionization, on a reasonable measuring time scale. Coincidence detection is necessary in order to identify outgoing electrons from the same ionization event, since they have an inherent energy relation.

New measurement techniques like DIPES call for concomitant consideration and further development of theory and computational tools to unravel its purport and interpretation. In one of our recent works, we reported a study of corevalence double photoionization of the $\mathrm{CS}_{2}$ molecule [6] where we applied a self-consistent field procedure for correlating complete or restricted active spaces. The optimization was based on a norm extended optimization in a reduced variational space where the core electron occupation was restricted to single occupation (restricted active space) and where the corresponding core orbital is frozen. This is followed by a straight Newton-Raphson optimization step to the full variational, local core-hole minimum. The results in that study agree with the experimentally well-resolved $\mathrm{C} 1 s^{-1} v^{-1}$ and $\mathrm{S} 2 p^{-1} v^{-1}$ double ionization electron spectra. The latter spectrum could also be interpreted by a simple, semiempirical model relying on the S $2 p$ spin-orbit splitting and the ordinary valence photoelectron spectrum. The application of the restricted active space method is favorable from the point of view that it provides a flexible scheme going from a "singleconfiguration" description with the smallest possible spincoupled combination of determinants to large valence orbital spaces with complete or restricted electron distributions, in all cases fulfilling spatial and spin symmetry. In the present work we aim to further advance this description by applying perturbation theory on the multiconfigurational active space and correcting for the core correlations in the ground-state energy which are unaccounted for in the scheme above. The use of MCSCF $a b$ initio techniques, of course, limits the range of applicability to molecules with relatively few atoms. Envisioning experimental measurements also on extended molecular systems and clusters, we apply in the present study our recently derived spin restricted open-shell density functional theory [12] to the optimization of core-valence hole states and computation of transition energies.

Core-valence dicationic states can be obtained by absorption of a single, high-energy photon, not only by direct double photoionization but also by Auger decay of a core-hole state with energy above that of the dicationic state. In the present spectra of the OCS molecule, the C $1 s^{-1} v^{-1}$ states can derive 
from Auger decay of the $\mathrm{O} 1 s^{-1}$ state while the core-valence dicationic states $\mathrm{S} 2 p^{-1} v^{-1}$ can, in principle, be reached from both $\mathrm{O} 1 s^{-1}$ and $\mathrm{C} 1 s^{-1}$ states. Such multiatom Auger emission (MAAE) has some analogy with the multiatom resonant photoemission (MARPE) proposed a few years ago as an effect that could be at the same time element specific as X-ray absorption and structurally dependent as electron diffraction. The first measurements of MARPE were later on shown by Ref. [13] to be affected by a nonlinearity of the electron detector used, and the actual effect was found to be smaller than originally thought. A theoretical investigation [14] also predicted a relatively low intensity of the resonant process in comparison to the direct photoionization cross section. MAAE intensity, that also involves two core holes strongly localized on different atoms, should also be weak and structure dependent.

In order to give a quantitative prediction of the MAAE effect and compare the intensity of Auger decay to corevalence dicationic states with that to valence-valence dicationic states observed in normal Auger spectra, we have used an $a b$ initio approach to simulate the nonradiative decay processes $\mathrm{O} 1 s^{-1} \rightarrow \mathrm{C} 1 s^{-1} \mathrm{v}^{-1}, \mathrm{O} 1 s^{-1} \rightarrow \mathrm{S} 2 p^{-1} v^{-1}$, and $\mathrm{C} 1 s^{-1} \rightarrow$ $\mathrm{S} 2 p^{-1} v^{-1}$ in OCS. The method, in a two-step approximation, is based on the projection of the Auger electron orbital in the electronic continuum on $L^{2}$ basis functions and Stieltjes imaging (SI) $[15,16]$ for the Auger rate. More recently, this method was extended using multicenter basis sets of Gaussian functions [17] to general polyatomic molecules beyond the usually adopted one-center expansion which, of course, would not allow for the calculation of the MAAE and MARPE effects that are intrinsically dependent on two atomic sites.

With the present work we primarily seek to establish a computational protocol for total energies of core-valence doubly ionized states and their transition energies with respect to the ground state. We demonstrate our computational protocol for the core-valence double ionization energies of the OCS molecule, analyze the corresponding $\mathrm{O} 1 s^{-1} v^{-1}, \mathrm{C} 1 s^{-1} v^{-1}$, and $\mathrm{S} 2 p^{-1} v^{-1}$ states, and compare the numerical results with state-of-the-art DIPES measurements.

\section{EXPERIMENTAL DETAILS}

The experimental data were recorded at beamline U49/2PGM-2 [18] at BESSY-II in Berlin, using a 2.2-m-long magnetic-bottle time-of-flight electron spectrometer designed for multiparticle coincidence measurements $[5,19,20]$. The time-to-energy conversion was calibrated using the $\mathrm{Kr} 3 d$ photoelectron lines [21] recorded at several photon energies. The flight times of two electrons originating from the same ionization process were measured with a time reference set by the ionizing photon pulse of the ring $[5,22]$. With respect to the latter, the experimental investigations were performed when the storage ring was operated in single bunch mode, which provides 30-ps light pulses at an interpulse period of 800.5 ns [23].

Multielectron coincidence data were recorded at the photon energies 230, 370, and $620 \mathrm{eV}$, which are well above the thresholds for creation of the $\mathrm{S} 2 p, \mathrm{C} 1 s$, and $\mathrm{O} 1 s$ hole, respectively, along with a vacancy in a valence shell. The monochromator was set to a resolution of $0.3 \mathrm{eV}$ or better.
The energy resolution of the electron spectrometer goes from approximately $20 \mathrm{meV}$ at the lowest kinetic energies to a nearly constant numerical resolution of about 50 at high kinetic energies. OCS gas was obtained commercially with a stated purity of $>99 \%$. Its purity was verified by the recording of valence photoelectron spectra.

\section{THEORETICAL DETAILS}

\section{A. Calculation of the hole states}

As discussed in our previous work [6], there are some particularities associated with core-valence double hole states which have implications for their calculation and choice of computational procedure. Without restrictions a MCSCF wave function will collapse to a low-lying double hole state by orbital rotation and by doubly occupying the original core orbital. We apply a two-step, second-order optimization procedure for the core-valence states to avoid orbital collapse $[10,24]$, where an intermediate optimization step with the core orbital frozen brings the wave function to the local region, followed by a full variational step using a Newton-Raphson technique relaxing also the core orbital. In the configurational space we impose single occupancy restrictions for the core orbital as the single core hole is only weakly interacting with continua of valence hole states (or double valence hole states for DIPES). This is accomplished using the restricted active space (RAS) technology [25,26], where the RAS1 space accommodates the singly occupied core orbital, while RAS2 is used for complete electron distributions among valence levels, and the RAS3 space can be used for the distribution of a small number of electrons. The selection of the active spaces can be guided by the occupation numbers from Møller-Plesset theory [27].

This scheme was applied in our previous work to analyze the DIPES spectra of $\mathrm{CS}_{2}$. In the present work we analyze further corrections. One is the dynamical correlation which is left out from a complete active space that is designed to preferentially cope with valence, near-degenerate, electronic excitations. We include a correction by assuming $N$ electron valence space pertubation theory of second order (NEVPT2) [28-30] as implemented in the DALTON program suite [31]. For utilizing the NEVPT2 machinery in DIPES, perturbative correlation corrections of equal level need to be applied for the ground state and the core-valence doubly ionized states. There is no published code where such perturbative method is implemented for core-hole states, due to the restricted active space construction of the wave function. This can, however, be overcome by using the well-known $Z+1$ approximation to the core-hole site and then modeling the resonance valence electronic structure for the core-valence hole state in question. By applying the $Z+1$ approximation, which thus replaces a core hole by an additonal nuclear charge, to the corresponding complete-active-space (CAS) state we get an estimate of the perturbational (dynamic correlation) contribution to the DIPES transition according to

$$
E_{\mathrm{corr}}=\left(E_{\mathrm{PT}}^{Z+1}-E_{\mathrm{CAS}}^{Z+1}\right)-\left(E_{\mathrm{PT}}^{\mathrm{gs}}-E_{\mathrm{CAS}}^{\mathrm{gs}}\right) .
$$

In equation (1) $E_{\mathrm{PT}}^{Z+1}$ and $E_{\mathrm{PT}}^{\mathrm{gs}}$ are the perturbation corrected dicationic state and ground state energies, and $E_{\mathrm{CAS}}^{Z+1}$ and $E_{\mathrm{CAS}}^{\mathrm{gs}}$ 
are the corresponding complete-active-space self-consistentfield (CASSCF) energies, respectively. We note that this correction does not distinguish between singlet and triplet states.

The second type of correction refers to the correlation energy of the core electron that is ionized. As the core orbital is "inactive," that is, uncorrelated in the CAS or NEVPT2 schemes, the ground-state energy is artifically raised with respect to the final state with a core hole. The leading core electron correlation energy can be obtained by a series of MP2 calculations excluding in steps the core orbitals. Compact, uncontracted basis sets are required for the radial correlation, while a compact higherangular-momentum function is required for the angular correlation.

Density functional theory (DFT) was used to calculate the ionization potential as the difference between the ground-state energy and the excited-state energy calculated from the doubly ionized Kohn-Sham determinant in a high-spin triplet state. The excited state density is optimized to give a minimum energy by alternating between core and valence optimization to avoid variational collapse. At first sight this is to use DFT in a naïve manner or at best as the semiempirical HartreeFock theory it is in practice. However, the obtained results which agree very well with other correlated calculations are not without theoretical basis.

The initial formulation of DFT by Hohenberg, Kohn, and Sham [32,33] established a relationship between the groundstate energy and the ground-state particle density. There have since then been many extensions to this ground-state theory, e.g., by Mermin who established a similar relationship between the grand canonical potential and the equilibrium density [34] and by Gunnarsson and Lundqvist [35], who introduced spin-polarization and generalized DFT for excited states that are lowest in a given symmetry. An attempt at a more general formalism for excited states was presented by Theophilou [36] which was based on the subspace spanned by the lowest states and the sum of their densities. Other examples include multiplets (von Barth [37]), bound excited states (Valone and Capitani [38]), and non- $\nu$-representable densities (Englisch and Englisch [39]).

In particular, Perdew and Levy [40] showed that an extremum of the ground-state energy functional is indeed a stationary state density, although the opposite does not hold. There is an additional condition that is not proven in this work that our solution to the Kohn-Sham equations provides the extremum of the energy functional. Nevertheless, our naïve approach to core-hole states with DFT, which is, first, motivated by our physical insight for the problem at hand, does have a theoretical foundation.

\section{B. Multiatomic Auger emission (MAAE)}

Adopting the two-step model that describes the nonradiative decay as independent from the previous core ionization step, the Auger rate is given, according to Fermi's Golden rule (also known as Wentzel ansatz in the context of Auger decay), by the squared Hamiltonian matrix element between the initial (core-hole) state $\left.\left.\right|^{2} \Psi_{c_{1}}\right\rangle$ and the final (core-valence dicationic) singlet (S) or triplet (T) state $\left.\left.\right|^{1,3} \Psi_{c_{2}, v}\right\rangle$ coupled to a continuum orbital $\phi_{\epsilon_{r}}$ describing the emitted Auger electron:

$$
\Gamma_{c_{2}, v, S / T}=2 \pi\left|V_{c_{2}, v, S / T}\left(\epsilon_{r}\right)\right|^{2}
$$

with

$$
V_{c_{2}, v, S / T}\left(\epsilon_{r}\right)=\left\langle{ }^{2} \Psi_{c_{1}}|\hat{\mathcal{H}}|^{1,3} \Psi_{c_{2}, v} \phi_{\epsilon_{r}}\right\rangle
$$

and

$$
\epsilon_{r}={ }^{2} E_{c_{1}}-{ }^{1,3} E_{c_{2}, v} .
$$

In the independent particle, independent channel approximation for discrete and continuum electronic states and frozen orbital approximation, the Auger amplitude takes a simple expression in terms of two-electron integrals

$$
\begin{aligned}
V_{c_{2}, v(S)}\left(\epsilon_{r}\right) & =\left\langle{ }^{2} \Psi_{c_{1}}|\hat{\mathcal{H}}|^{2} \Psi_{c_{2}, v(S), \epsilon_{r}}\right\rangle \\
& =\sqrt{\frac{1}{2}}\left(\left[c_{2} c_{1} \mid v \epsilon_{r}\right]+\left[c_{2} \epsilon_{r} \mid v c_{1}\right]\right) \\
V_{c_{2}, v(T)}\left(\epsilon_{r}\right) & =\left\langle{ }^{2} \Psi_{c_{1}}|\hat{\mathcal{H}}|^{2} \Psi_{c_{2}, v(T), \epsilon_{r}}\right\rangle \\
& =\sqrt{\frac{3}{2}}\left(\left[c_{2} c_{1} \mid v \epsilon_{r}\right]-\left[c_{2} \epsilon_{r} \mid v c_{1}\right]\right),
\end{aligned}
$$

while the continuum orbital $\phi_{\epsilon_{r}}$ can be computed by diagonalization of a channel static-exchange Hamiltonian projected onto a large Gaussian basis set. The SI procedure provides a smooth amplitude distribution versus the continuum energy that can be easily interpolated at the resonance energy $\epsilon_{r}$; more details can be found in Refs. [16,17].

\section{COMPUTATIONAL DETAILS}

The geometry of the OCS molecule was assumed linear with $\mathrm{O}-\mathrm{C}$ and C-S distances of $1.1239 \AA$ and $1.5640 \AA$, respectively. Vibrational excitations responsible for the band broadening were neglected and the calculations utilized $C_{2 v}$ point group symmetry (the real symmetry being $C_{\infty \nu}$ ). The dicationic states with $9 \sigma$ and $8 \sigma$ orbital vacancies, and $3 \pi$ and $2 \pi$ vacancies, appear as the two lowest doubly ionized states (with correct core hole) in $\Sigma$ and $\Pi$ symmetry. To study systematically the different electronic structure methods, the computations were made for these four valence vacancies and the three core holes in four different schemes, denoted by A, B, C, and D.

The MCSCF calculations were performed in three different schemes, A, B, and C, where A refers to MCSCF optimization using the restricted active space technique. In scheme $B$ the energies were obtained from those of scheme A by adding full valence correlation from the NEVPT2 perturbation theory in the $Z+1$ approximation, as outlined above. In cases $A$ and $\mathrm{B}$, the CASSCF and the restricted active-space self-consistent field (RASSCF) states were optimized using active spaces $(5,4,4,1)$ with the inactive space $(7,1,1,0)$ corresponding to the $\mathrm{O} 1 s, \mathrm{O} 2 s, \mathrm{C} 1 s, \mathrm{C} 2 s, \mathrm{~S} 1 s, \mathrm{~S} 2 s$, and $\mathrm{S} 2 p$ orbitals. In scheme $\mathrm{C}$ the core relaxation was modeled using single configuration wave functions, and the valence orbital involved in the formation of the dicationic state was fixed with a single electron occupation using the RAS3 space technique.

The NEVPT2 correction for the valence correlation was calculated for the ground state and the doubly ionized state. In scheme $\mathrm{B}$ the core hole was modeled in the $Z+1$ 
TABLE I. Ground-state MP2 corrections (in eV) to account for core-core correlation. For details, see text.

\begin{tabular}{lcc}
\hline \hline $\mathrm{C} 1 s$ & $\mathrm{O} 1 s$ & $\mathrm{~S} 2 p$ \\
\hline-1.330 & -1.481 & -1.792 \\
\hline \hline
\end{tabular}

approximation and the correction using NEVPT2 on CAS $(4,3,3,1)$ calculation with the same $(7,1,1,0)$ inactive space was then applied to calculation A.

For calculations of the MP2 correction of the ground-state core correlation, all contractions were removed from the basis set. In addition, tight $p$ functions with exponents 80.0, 100.0, and 1200.0 on $\mathrm{C}, \mathrm{O}$, and $\mathrm{S}$, respectively, were added to the basis set. Stepwise release of the orbitals, subject to MP2 perturbation, produced correlation energies related to each core orbital. The results are presented in Table I and are included in the presented values of the IPs and DIPs of methods A-C.

The last calculation scheme D utilized DFT with the B3LYP exchange-correlation functional. The calculation was based on the two-step relaxation process of the single core-hole state. Doubly ionized states were then obtained by freezing the core orbital and the valence orbital with fixed unity occupation. Other functionals (LDA, BP86, PBE, BLYP, CAMB3LYP, $\mathrm{B} 3 \mathrm{LYP}$ ) were also used, producing consistent results (usually in an $\mathrm{eV}$ from each other), except for LDA which gave double ionization energies that were several eVs off for the $\mathrm{C} 1 \mathrm{~s}$ and O $1 s$ core-hole states.

All MCSCF calculations were performed by the molecular electronic structure program DALTON (release 2.0 (2005) [31]). The open-shell DFT part was carried out by a local unreleased version of DALTON. Following previous basis set investigations on the $\mathrm{CS}_{2}$ molecule [6], the calculations were performed utilizing the augmented correlation-consistent polarized corevalence triple zeta (aug-cc-pCVTZ) [41,42] basis set.

\section{RESULTS AND DISCUSSION}

\section{A. O1s-valence DIPES}

Figure 1 shows the $\mathrm{O} 1 s^{-1} v^{-1}$ DIPES, measured using the photon energy of $620 \mathrm{eV}$, along with the energies obtained from the calculations A-D. As can be seen, the $\mathrm{O} 1 s^{-1}(v)^{-1}$ spectrum has an onset at around $560 \mathrm{eV}$, which is about $20 \mathrm{eV}$ above the $\mathrm{O} 1 s$ single ionization energy at $540.3 \mathrm{eV}$ (cf. Ref. [43]). In the recorded energy region, a structure of

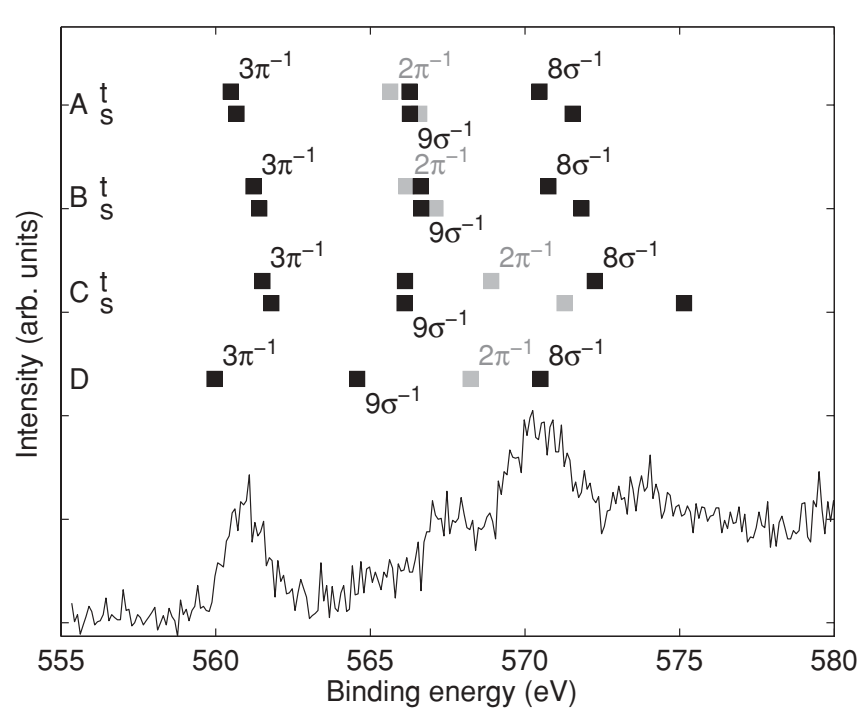

FIG. 1. The experimental $\mathrm{O} 1 \mathrm{~s}^{-1}(\mathrm{v})^{-1}$ core-valence double ionization electron spectrum of the OCS molecule recorded at the photon energy of $620 \mathrm{eV}$. Core-valence double ionization energies obtained from calculations A-D are shown above the experimental spectrum. Values from methods $\mathrm{A}-\mathrm{C}$ include the ground-state MP2 correction of $+1.481 \mathrm{eV}$. To make the figure easier to comprehend, we have used, where necessary for distinction, black and gray markers as well as black and gray labels correspondingly. For more details, see text.

four peaks is seen between 560 and $575 \mathrm{eV}$, where that at around $571 \mathrm{eV}$ is the most intense.

The calculated energies for the four core-valence hole states are also listed in Table II. Generally, the calculated energies show small singlet-triplet splittings and invariance for the energies of the $\mathrm{O} 1 s^{-1} 3 \pi^{-1}$ and $\mathrm{O} 1 s^{-1} 9 \sigma^{-1}$ states with respect to the method used. These states are the lowest in their symmetry with the $\mathrm{O} 1 s$ core hole in the $C_{2 v}$ point group. In contrast, the excited states $\left(\mathrm{O} 1 s^{-1} 8 \sigma^{-1}\right.$ and $\left.\mathrm{O} 1 s^{-1} 2 \pi^{-1}\right)$ show a variety of energies depending on the calculation method used. The small singlet-triplet splittings originate from small exchange integrals and weak correlation between the core and valence holes, as expected from our previous study on $\mathrm{CS}_{2}$ [6]. The splittings in the calculations are smallest for the lowest states $\mathrm{O} 1 s^{-1} 3 \pi^{-1}$ and $\mathrm{O} 1 s^{-1} 9 \sigma^{-1}$.

Without the MP2 correction, the calculation scheme A locates the $\mathrm{O} 1 s^{-1}$ single ionization energy at $539.59 \mathrm{eV}$, which is $0.71 \mathrm{eV}$ less than the experimental value [43]. When the MP2 correction is applied, the energy is overestimated by

TABLE II. Calculated $\mathrm{O} 1 s^{-1} v^{-1}$ double ionization energies (in $\mathrm{eV}$ ). For comparison, the single core ionization energies are given for schemes A and D (the corresponding experimental value is $540.3 \mathrm{eV}$ (cf. Ref. [43]). Values from MCSCF calculations $\mathrm{A}-\mathrm{C}$ include the ground-state MP2 correction of $+1.481 \mathrm{eV}$ for core-core correlation. Details can be found in the text.

\begin{tabular}{|c|c|c|c|c|c|c|c|}
\hline \multirow[b]{2}{*}{ Configuration } & \multicolumn{2}{|c|}{ A } & \multicolumn{2}{|c|}{ B } & \multicolumn{2}{|c|}{$\mathrm{C}$} & \multirow[b]{2}{*}{$\mathrm{D}$} \\
\hline & Singlet & Triplet & Singlet & Triplet & Singlet & Triplet & \\
\hline $\mathrm{O} 1 s^{-1}$ & \multicolumn{2}{|c|}{541.069} & \multicolumn{2}{|c|}{-} & \multicolumn{2}{|c|}{ - } & 540.084 \\
\hline $\mathrm{O} 1 s^{-1} 3 \pi^{-1}$ & 560.672 & 560.487 & 561.408 & 561.224 & 561.801 & 561.511 & 559.964 \\
\hline $\mathrm{O} 1 s^{-1} 2 \pi^{-1}$ & 566.582 & 565.643 & 567.105 & 566.167 & 571.281 & 568.911 & 568.244 \\
\hline $\mathrm{O} 1 s^{-1} 9 \sigma^{-1}$ & 566.281 & 566.271 & 566.646 & 566.636 & 566.111 & 566.121 & 564.573 \\
\hline $\mathrm{O} 1 s^{-1} 8 \sigma^{-1}$ & 571.543 & 570.473 & 571.826 & 570.756 & 575.141 & 572.261 & 570.502 \\
\hline
\end{tabular}


TABLE III. O $1 s^{-1} \mathrm{v}^{-1}$ double ionization energies (in $\mathrm{eV}$ ) obtained using some selected exchange-correlation functionals for DFT. The B3LYP energies are presented for comparison. For reference, the experimental O $1 s$ single ionization energy is at $540.3 \mathrm{eV}$ (cf. Ref. [43]).

\begin{tabular}{lcccccc}
\hline \hline Configuration & LDA & BP86 & PBE0 & BLYP & camB3LYP & B3LYP \\
\hline $\mathrm{O} 1 s^{-1}$ & 535.426 & 540.073 & 539.710 & 540.040 & 540.301 & 540.084 \\
$\mathrm{O} 1 s^{-1} 3 \pi^{-1}$ & 555.606 & 560.091 & 559.670 & 559.820 & 560.251 & 559.964 \\
$\mathrm{O} 1 s^{-1} 2 \pi^{-1}$ & 563.866 & 568.184 & 567.949 & 567.937 & 568.679 & 568.244 \\
$\mathrm{O} 1 s^{-1} 9 \sigma^{-1}$ & 559.925 & 564.496 & 564.277 & 564.250 & 564.940 & 564.573 \\
$\mathrm{O} 1 s^{-1} 8 \sigma^{-1}$ & 566.167 & 570.477 & 570.211 & 570.227 & 570.944 & 570.502 \\
\hline \hline
\end{tabular}

about the same amount. The $\mathrm{O} 1 s^{-1} 3 \pi^{-1}$ energy with MP2 correction, however, is located right at the first line in the DIPES of Fig. 1. According to scheme A, the $\mathrm{O} 1 s^{-1} 2 \pi^{-1}$ and $\mathrm{O} 1 s^{-1} 9 \sigma^{-1}$ states overlap at $566 \mathrm{eV}$. In addition, the highest state, $\mathrm{O} 1 \mathrm{~s}^{-1} 8 \sigma^{-1}$ is obtained at $570 \mathrm{eV}$ in agreement with the most intense line in the experimental spectrum in Fig. 1.

The $(4,3,3,1)$ NEVPT2 corrections [cf. Eq. (1)] to the $\mathrm{O} 1 s^{-1} \mathrm{v}^{-1}$ DIPs were relatively small, the maximum being $0.74 \mathrm{eV}$ for the $\mathrm{O} 1 s^{-1} 3 \pi^{-1}$ state. Even though the groundstate correction and the excited state corrections were several $\mathrm{eVs}$, they cancel each other to a large degree and result in a modest correction to the DIP. All NEVPT2 corrections resulted in larger ionization energies, which could be understood as stronger dynamical correlation in the neutral ground state than in the doubly ionized state of the OCS molecule by virtue of having an extra valence electron.

The calculation scheme $\mathrm{C}$ gives an even higher $\mathrm{O} 1 \mathrm{~s}^{-1} 3 \pi^{-1}$ energy than schemes $\mathrm{A}$ and $\mathrm{B}$. In this case the double ionization energy is slightly overestimated. Method $\mathrm{C}$ relies on $\mathrm{HF}$ optimization of the singly core ionized states, which appears to allow less relaxation for the orbitals as the DIPs are higher than the values obtained from MCSCF methods A and B, except for the $\mathrm{O} 1 s^{-1} 9 \sigma^{-1}$ state. Method $\mathrm{C}$ also results in larger singlet-triplet splittings for the excited states $\mathrm{O} 1 s^{-1} 8 \sigma^{-1}$ and $\mathrm{O} 1 s^{-1} 2 \pi^{-1}$.

Scheme D, utilizing B3LYP-DFT, locates the O $1 s$ single ionization energy at $540.084 \mathrm{eV}$, which is about $0.22 \mathrm{eV}$ below the experimental value [43]. The DIPES energies are somewhat lower than for the MP2 corrected MCSCF methods A-C. The excited $\mathrm{O} 1 s^{-1} 2 \pi^{-1}$ and $\mathrm{O} 1 s^{-1} 8 \sigma^{-1}$ states are also lower than the corresponding singlet and triplet states from the method C. A series of DFT calculations using different exchange-correlation functionals were performed and the DIPs obtained are presented in Table III. As can be seen, different functionals give rather similar results, save for LDA. One possible origin of this discrepancy is the complete lack of Hartree-Fock exchange in the LDA functional, which leads to a larger self-interaction error and also to a poorer distribution of the core electron density. Even a very slight change in the core density has pronounced energy effects. Moreover, the DFT self-interaction error is comparatively large for core electrons [44].

\section{B. C1s-valence DIPES}

The $\mathrm{C} 1 s^{-1}(\mathrm{v})^{-1}$ DIPES, measured at the photon energy of $370 \mathrm{eV}$, is shown in Fig. 2 along with the energies obtained from calculations A-D. The lowest C $1 s$-valence double ionization energy is observed at around $315 \mathrm{eV}$ and a comparatively strong peak is observed at $321 \mathrm{eV}$ with a possible shoulder on the higher binding energy side.

The calculated energies for the core-valence ionized states are presented in Table IV. As in the case of the O 1s core hole, the singlet-triplet splitting for the $\mathrm{C} 1 s^{-1}(v)^{-1}$ states is small. Two opposite trends of the double ionization energies as a function of the computational scheme is observed. The $\pi^{-1}$ states move up in energy when proceeding from method A to $\mathrm{C}$, while the $\sigma^{-1}$ states move down in energy, especially when the $\mathrm{HF}$ core relaxation method $\mathrm{C}$ with valence unity occupation is used. The DFT results agree very well with the experiment.

Without the MP2 correction calculation A reproduces the C 1s single ionization energy quite well as does calculation D too. These methods give values of 295.227 and $295.525 \mathrm{eV}$, respectively, which differ by some 0.03 and $0.33 \mathrm{eV}$ from the experimental value of $295.20 \mathrm{eV}$ [43]. When the MP2 correction is applied in scheme A, the single ionization energy

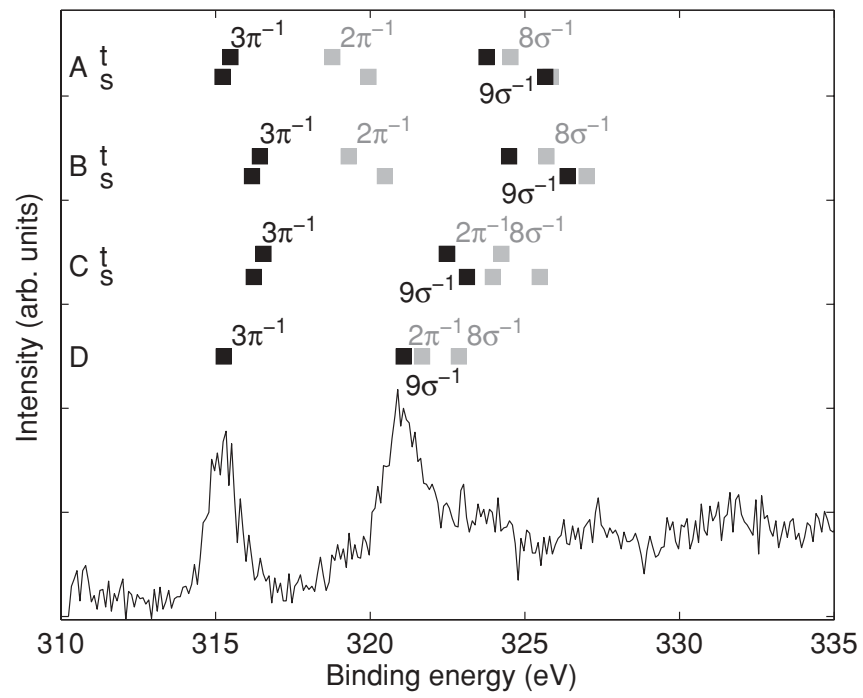

FIG. 2. The experimental $\mathrm{C} 1 s^{-1} \mathrm{v}^{-1}$ core-valence double ionization electron spectrum of OCS recorded at the photon energy of $370 \mathrm{eV}$. Core-valence double ionization energies obtained from calculations A-D are shown above the experimental spectrum. Values from methods A-C include the ground-state MP2 correction of $+1.330 \mathrm{eV}$. To make the figure easier to comprehend, we have used, where necessary for distinction, black and gray markers as well as black and gray labels correspondingly. For more details, see text. 
TABLE IV. Calculated C $1 s^{-1} v^{-1}$ double ionization energies (in eV). For comparison, the single core ionization energies are given for schemes A and D (the corresponding experimental value is $295.20 \mathrm{eV}$ [43]). Values from MCSCF calculations A-C include the ground-state MP2 correction of $+1.330 \mathrm{eV}$ for core-core correlation. More details are given in the text.

\begin{tabular}{|c|c|c|c|c|c|c|c|}
\hline \multirow[b]{2}{*}{ Configuration } & \multicolumn{2}{|c|}{ A } & \multicolumn{2}{|c|}{ B } & \multicolumn{2}{|c|}{$\mathrm{C}$} & \multirow[b]{2}{*}{$\mathrm{D}$} \\
\hline & Singlet & Triplet & Singlet & Triplet & Singlet & Triplet & \\
\hline $\mathrm{C} 1 s^{-1}$ & \multicolumn{2}{|c|}{296.557} & \multicolumn{2}{|c|}{ - } & \multicolumn{2}{|c|}{ - } & 295.525 \\
\hline $\mathrm{C} 1 s^{-1} 3 \pi^{-1}$ & 315.230 & 315.485 & 316.179 & 316.434 & 316.240 & 316.550 & 315.267 \\
\hline $\mathrm{C} 1 s^{-1} 2 \pi^{-1}$ & 319.947 & 318.773 & 320.479 & 319.305 & 323.970 & 322.500 & 321.676 \\
\hline $\mathrm{C} 1 s^{-1} 9 \sigma^{-1}$ & 325.659 & 323.765 & 326.387 & 324.493 & 323.130 & 322.480 & 321.084 \\
\hline $\mathrm{C} 1 s^{-1} 8 \sigma^{-1}$ & 325.833 & 324.535 & 326.997 & 325.699 & 325.480 & 324.240 & 322.865 \\
\hline
\end{tabular}

is overestimated. When comparing the double ionization energies according to scheme A, the MP2 corrected $\mathrm{C} 1 s^{-1} 3 \pi^{-1}$ binding energy is in good agreement with the experiment whilst the $8 \sigma^{-1}$ and $9 \sigma^{-1}$ states are too high in energy. The $\mathrm{C} 1 s^{-1} 2 \pi^{-1}$ states, on the other hand, are shifted toward lower energies, as can be seen in Fig. 2.

The $(4,3,3,1)$ NEVPT2 correction for $\mathrm{C} 1 s$ is not large. The maximum value of the corrections, all positive, was $1.16 \mathrm{eV}$ for $\mathrm{C} 1 s^{-1} 8 \sigma^{-1}$ states. The corrections do not lead to any change in the interpretation of the spectrum. Scheme $\mathrm{C}$ with HF relaxation and fixed valence orbital occupation reproduces the lowest $\mathrm{C} 1 s^{-1} 9 \sigma^{-1}$ states better but is still somewhat overestimating their DIPs. In contrast to methods A and B, method $C$ results in a gathering of the three states other than $\mathrm{C} 1 s^{-1} 3 \pi^{-1}$ in the region 322 to $325 \mathrm{eV}$, somewhat beyond the second large peak in the spectrum of Fig. 2 .

The DFT scheme D gives values similar to scheme C, shifted slightly to lower binding energies. According to this method the $\mathrm{C} 1 s^{-1} 3 \pi^{-1}$ states reproduce the peak at $315 \mathrm{eV}$ and the $\mathrm{C} 1 s^{-1} 2 \pi^{-1}$ and $\mathrm{C} 1 s^{-1} 9 \sigma^{-1}$ the peak at $321.5 \mathrm{eV}$. Furthermore the configuration $\mathrm{C} 1 s^{-1} 8 \sigma^{-1}$ seems to explain the high energy shoulder at around $324 \mathrm{eV}$.

The results of different DFT functionals are presented in Table V. A good agreement between the different functionals is again obtained, except for LDA. The CAMB3LYP functional is seen to give the highest double ionization energies. A possible reason for this behavior of the LDA was discussed in the previous section on the oxygen spectrum.

\section{S $2 p$-valence DIPES}

The S $2 p^{-1} v^{-1}$ DIPES is complicated due to the splitting induced by spin-orbit interaction of the core hole, which is expected to create a double structure in the DIPES. In our nonrelativistic calculations, the spin-orbit splitting, according to Ref. [6], was taken into account by diagonalization of a perturbation matrix. Another complication is the molecular field splitting of the S $2 p$ orbitals which, however, is very small and has been omitted. The MCSCF methods A-C were chosen for the non-spin-orbit-perturbed energies and resulted in singlet and triplet states.

The S $2 p^{-1} v^{-1}$ DIPES, measured at the photon energy of $230 \mathrm{eV}$, is shown in Fig. 3 along with the calculated energies which are also given in Table VI. The spectrum starts at $192 \mathrm{eV}$ with an intense double peak structure due to spin-orbit splitting. The remaining features of the spectrum are double line structures in the 195- to $197-\mathrm{eV}$ region with opposite intensity distribution and two peaks with not so clear substructures.

According to scheme A without MP2 correction, and including the spin-orbit perturbation, the $\mathrm{S} 2 p_{3 / 2}$ single ionization energy is at $169.62 \mathrm{eV}$, which is about $1 \mathrm{eV}$ less than the experimental value of $170.60 \mathrm{eV}$ (cf. Ref. [43]). The MP2 correction for the ground-state excess core correlation was found to be $+1.79 \mathrm{eV}$, which accounts for most of this discrepancy. The numerical DIPES obtained by method A is, however, slightly shifted toward higher binding energies. Scheme A suggests that the $\mathrm{S} 2 p^{-1} 3 \pi^{-1}$ character is assigned to the lowest two peaks and S $2 p^{-1} 2 \pi^{-1}$ to the next two peaks. It is noteworthy that the $\mathrm{S} 2 p^{-1} 8 \sigma^{-1}$ states are well above the ionization energy of $200 \mathrm{eV}$, where essentially no lines are seen in the experimental spectrum.

According to previous discussions, it is not surprising that the NEVPT2 corrected scheme $\mathrm{B}$ gives results similar to scheme A. The largest correction for the S $2 p$ DIPES was $1.26 \mathrm{eV}$ and the smallest $0.64 \mathrm{eV}$, all positive in sign.

TABLE V. C $1 s^{-1} \mathrm{v}^{-1}$ double ionization energies (in $\mathrm{eV}$ ) obtained using some selected exchange-correlation functionals for DFT. The B3LYP energies are presented for comparison. The experimental C1s single ionization energy is $295.20 \mathrm{eV}$ (cf. Ref. [43]).

\begin{tabular}{lcccccr}
\hline \hline Configuration & LDA & BP86 & PBE0 & BLYP & camB3LYP & B3LYP \\
\hline $\mathrm{C} 1 s^{-1}$ & 291.541 & 294.851 & 295.161 & 294.952 & 295.847 & 295.53 \\
$\mathrm{C} 1 s^{-1} 3 \pi^{-1}$ & 311.606 & 314.750 & 314.973 & 314.614 & 315.640 & 315.27 \\
$\mathrm{C} 1 s^{-1} 2 \pi^{-1}$ & 317.837 & 320.855 & 321.449 & 320.731 & 322.325 & 321.68 \\
$\mathrm{C} 1 s^{-1} 9 \sigma^{-1}$ & 316.863 & 320.125 & 320.825 & 320.018 & 321.687 & 321.08 \\
$\mathrm{C} 1 s^{-1} 8 \sigma^{-1}$ & 318.656 & 322.017 & 322.681 & 321.829 & 323.447 & 322.87 \\
\hline \hline
\end{tabular}




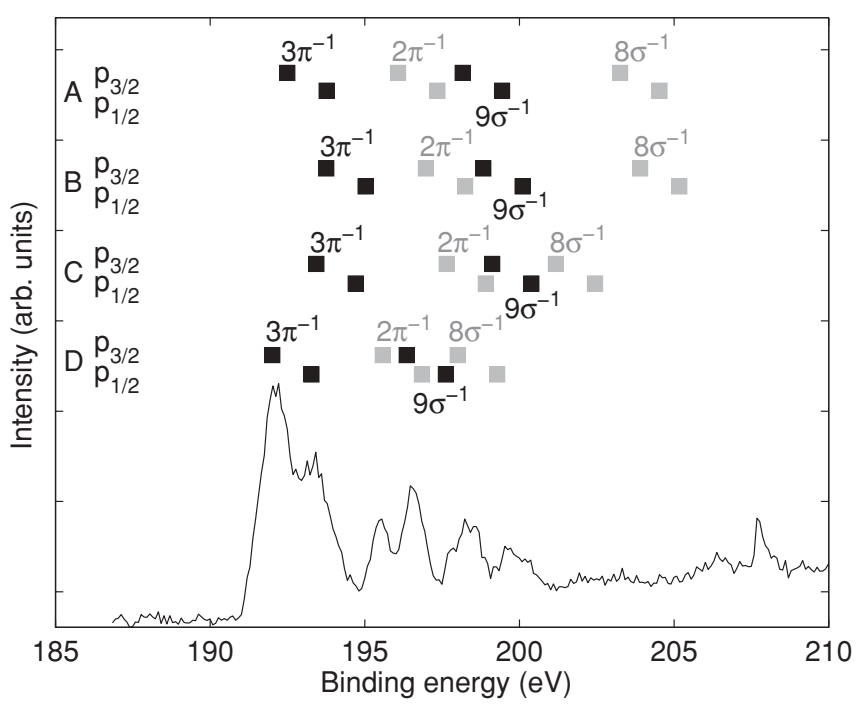

FIG. 3. The experimental S $2 p^{-1} \mathrm{v}^{-1}$ core-valence double ionization electron spectrum of the OCS molecule recorded at the photon energy of $230 \mathrm{eV}$. Core-valence double ionization energies obtained from the calculations A-D are shown above the experimental spectrum. Values from methods A-C include the ground-state MP2 correction of $+1.79 \mathrm{eV}$. To make the figure easier to comprehend, we have used, where necessary for distinction, black and gray markers as well as black and gray labels correspondingly. For more details, see text.

The effect of the correction is some overestimation of the double ionization energies. Both methods A and B suggest that the experimental S $2 p^{-1} 2 \pi^{-1}$ line components may not have the statistical intensity distribution $2: 1$ as the $S 2 p_{1 / 2}^{-1} 2 \pi^{-1}$ and $S 2 p_{3 / 2}^{-1} 9 \sigma^{-1}$ transitions overlap in energy. The NEVPT2 correction does not describe the $\mathrm{S} 2 p^{-1} 8 \sigma^{-1}$ states any better and the peak at around $200 \mathrm{eV}$ remains unexplained by schemes A and B.

So far, the performance of scheme $\mathrm{C}$ has differed from that of the MCSCF schemes A and B, and so it does also in the case of the $S 2 p^{-1} \mathrm{~V}^{-1}$ DIPES. Despite some error in the $\mathrm{S} 2 p^{-1} 3 \pi^{-1}, \mathrm{~S} 2 p^{-1} 2 \pi^{-1}$, and $\mathrm{S} 2 p^{-1} 9 \sigma^{-1}$ states, the $\mathrm{S} 2 p^{-1} 8 \sigma^{-1}$ transitions are lower in energy than in schemes A and B. In contrast, scheme D with DFT predicts well the two lowest double peak structures which assigns them to $\mathrm{S} 2 p^{-1} 3 \pi^{-1}$ and S $2 p^{-1} 2 \pi^{-1}$ states. The S $2 p^{-1} 9 \sigma^{-1}$ states are located at somewhat lower energies than in other methods, and the $\mathrm{S} 2 p^{-1} 8 \sigma^{-1}$ states coincide almost with the two highest peaks just below $200 \mathrm{eV}$. The inverted intensity of the $\mathrm{S} 2 p 2 \pi^{-1}$ lines is explained by the calculation D as an overlap between the S $2 p_{1 / 2}^{-1} 2 \pi^{-1}$ and S $2 p_{3 / 2}^{-1} 9 \sigma^{-1}$ states.

Double ionization energies obtained using different DFT functionals are presented in Table VII. A consistent performance is seen between different functionals. It is noteworthy that unlike in the previous cases of $\mathrm{C} 1 s$ and $\mathrm{O} 1 s$ core holes, for $\mathrm{S} 2 p$ hole states the LDA functional agrees within marginals of around $1 \mathrm{eV}$ to the other functionals. This might be related to the less shallow nature of the $\mathrm{S} 2 p$ density which is better described by DFT with Hartree-Fock exchange in comparison to the deeper $\mathrm{C} 1 s$ and $\mathrm{O} 1 s$ densities.

To conclude this section, the clearest trend seen between the different methods is that schemes A-C give somewhat too-high energies of the S $2 p^{-1} 3 \pi^{-1}, \mathrm{~S} 2 p^{-1} 2 \pi^{-1}$, and S $2 p^{-1} 9 \sigma^{-1}$ states. The DIP of the S $2 p^{-1} 8 \sigma^{-1}$ state seems to depend on the method applied, i.e., A, B, or C. Overestimation of the energies may be due to exclusion of the three- and four-body terms in the MP2 correlation correction, which has proven to work better for nondegenerate orbitals. The DFT method D works well, allowing a one-to-one assignment, practically without any energy shift. Also different DFT functionals seem to work consistently, even in LDA, which, in contrast, failed for the cases of $\mathrm{O} 1 s$ and $\mathrm{C} 1 s$ core hole states.

\section{MAAE results}

Figure 4 shows the computed $\mathrm{O} 1 s^{-1} \rightarrow \mathrm{C} 1 s^{-1} v^{-1}$ corevalence Auger spectrum of the OCS molecule, where each label indicates the valence hole and black and gray bars correspond to singlet and triplet coupling of the core-valence holes, respectively. From an energetic point of view it can be observed that the splitting associated with spin-spin coupling, which is proportional to the exchange integral $\left[v c_{2} \mid c_{2} v\right]$, depends on the penetration of the valence orbital into the core region. In contrast to a standard (valence-valence) Auger spectrum, the intensities in Fig. 4 show dominating triplet states with a triplet-to-singlet intensity ratio very close to the statistical ratio; this is due to the fact that the integral [C $\left.1 s \epsilon_{r} \mid v \mathrm{O} 1 s\right]$ in Eqs. (5) and (6) is about one order of magnitude larger than the integral $\left[\mathrm{C} 1 s \mathrm{O} 1 s \mid v \epsilon_{r}\right]$. Another set of dicationic core-valence states that could, in principle, be reached by the nonradiative decay of the $\mathrm{O} 1 s$ hole are the $\mathrm{S} 2 p^{-1} v^{-1}$. Our calculations (not shown) predict for this Auger

TABLE VI. Calculated S $2 p^{-1} \mathrm{v}^{-1}$ double ionization energies (in eV). For comparison, the single core ionization energies are given for schemes $\mathrm{A}$ and $\mathrm{D}$ (the lowest experimental single ionization energy corresponding to the $\mathrm{S} 2 p_{3 / 2}$ component is $170.60 \mathrm{eV}$ (cf. Ref. [43])). Values from MCSCF calculations A-C include the ground-state MP2 correction of $+1.79 \mathrm{eV}$ for core-core correlation. More details can be found in the text.

\begin{tabular}{|c|c|c|c|c|c|c|c|c|}
\hline \multirow[b]{2}{*}{ Configuration } & \multicolumn{2}{|c|}{ A } & \multicolumn{2}{|c|}{$\mathrm{B}$} & \multicolumn{2}{|r|}{$\mathrm{C}$} & \multicolumn{2}{|c|}{$\mathrm{D}$} \\
\hline & $p_{3 / 2}$ & $p_{1 / 2}$ & $p_{3 / 2}$ & $p_{1 / 2}$ & $p_{3 / 2}$ & $p_{1 / 2}$ & $p_{3 / 2}$ & $p_{1 / 2}$ \\
\hline $\mathrm{S} 2 p_{3 / 2}^{-1}$ & 171.412 & & & & & - & 170.545 & \\
\hline $\mathrm{S} 2 p^{-1} 3 \pi^{-1}$ & 192.499 & 193.769 & 193.759 & 195.029 & 193.439 & 194.708 & 192.008 & 193.278 \\
\hline $\mathrm{S} 2 p^{-1} 2 \pi^{-1}$ & 196.079 & 197.349 & 196.978 & 198.248 & 197.649 & 198.918 & 195.584 & 196.853 \\
\hline $\mathrm{S} 2 p^{-1} 9 \sigma^{-1}$ & 198.171 & 199.441 & 198.834 & 200.105 & 199.119 & 200.388 & 196.357 & 197.627 \\
\hline $\mathrm{S} 2 p^{-1} 8 \sigma^{-1}$ & 203.261 & 204.530 & 203.900 & 205.170 & 201.179 & 202.448 & 198.010 & 199.280 \\
\hline
\end{tabular}


TABLE VII. S $p^{-1} v^{-1}$ double ionization energies (in $\mathrm{eV}$ ) obtained using some selected exchange-correlation functionals for DFT. The B3LYP energies are presented for comparison.

\begin{tabular}{lcccccc}
\hline \hline Configuration & LDA & BP86 & PBE0 & BLYP & camB3LYP & B3LYP \\
\hline $\mathrm{S} 2 p_{3 / 2}^{-1}$ & 170.756 & 170.228 & 170.435 & 170.095 & 170.565 & 170.545 \\
$\mathrm{~S} 2 p_{3 / 2}^{-1} 3 \pi^{-1}$ & 192.353 & 191.696 & 192.018 & 191.318 & 192.205 & 192.008 \\
$\mathrm{~S} 2 p_{3 / 2}^{-1} 2 \pi^{-1}$ & 195.872 & 195.102 & 195.626 & 194.737 & 195.980 & 195.584 \\
$\mathrm{~S} 2 p_{3 / 2}^{-1} 9 \sigma^{-1}$ & 196.821 & 196.077 & 196.350 & 195.726 & 196.692 & 196.357 \\
$\mathrm{~S} 2 p_{3 / 2}^{-1} 8 \sigma^{-1}$ & 198.123 & 197.513 & 198.112 & 197.125 & 198.418 & 198.010 \\
$\mathrm{~S} 2 p_{1 / 2}^{-1}$ & 172.026 & 171.498 & 171.705 & 171.365 & 171.835 & 171.815 \\
$\mathrm{~S} 2 p_{1 / 2}^{-1} 3 \pi^{-1}$ & 193.623 & 192.966 & 193.288 & 192.588 & 193.475 & 193.278 \\
$\mathrm{~S} 2 p_{1 / 2}^{-1} 2 \pi^{-1}$ & 197.142 & 196.372 & 196.896 & 196.007 & 197.250 & 196.853 \\
$\mathrm{~S} 2 p_{1 / 2}^{-1} 9 \sigma^{-1}$ & 198.091 & 197.347 & 197.620 & 196.996 & 197.962 & 197.627 \\
$\mathrm{~S} 2 p_{1 / 2}^{-1} 8 \sigma^{-1}$ & 199.393 & 198.783 & 199.382 & 198.395 & 199.688 & 199.280 \\
\hline \hline
\end{tabular}

spectrum intensities about two orders of magnitude smaller than the ones in Fig. 4. This reflects the strong dependence of the MAAE effect on the distance among the core sites, which for O-S is about twice as large as that for O-C; an exponential dependence on the distance was previously predicted for the MARPE effect by calculations on CO [14].

The dicationic states $\mathrm{S} 2 p^{-1} v^{-1}$ can be reached more easily by the decay process $\mathrm{C} 1 s^{-1} \rightarrow \mathrm{S} 2 p^{-1} v^{-1}$; the corresponding computed core-valence Auger spectrum is shown in Fig. 5. In this case, the singlet and triplet intensities, obtained from calculations not explicitly including the spin-orbit splitting, have been statistically distributed over the $2 p_{3 / 2}$ and $2 p_{1 / 2}$ deriving peaks whose energy has been obtained from method $\mathrm{C}$, as was discussed in the previous section.

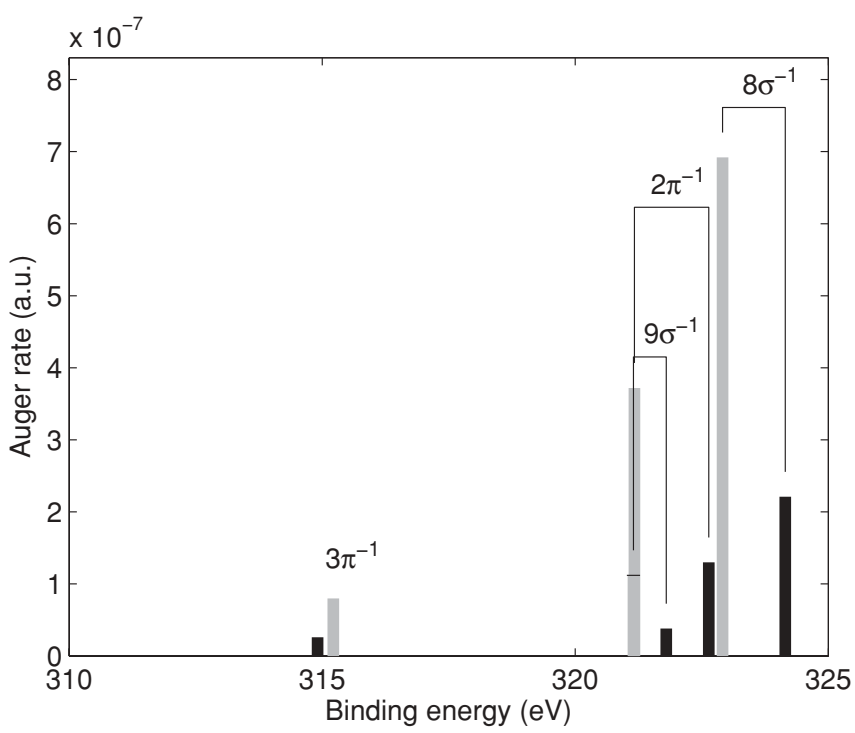

FIG. 4. The computed $\mathrm{O} 1 s^{-1} \rightarrow \mathrm{C} 1 s^{-1} \mathrm{v}^{-1}$ core-valence Auger spectrum of the OCS molecule. The energies were obtained from MCSCF calculations and the intensities (atomic units) from the SI procedure in the independent particle, independent channel approximation. The singlet states are shown in black and the triplet states are shown in gray.
For comparison with the numerical core-valence spectra in Figs. 4 and 5, Fig. 6 shows the low energy part of the $\mathrm{O} 1 s^{-1} \rightarrow v_{1}^{-1} v_{2}^{-1}$ valence-valence Auger spectrum of OCS where the intensities have been obtained by the same method as described previously, while the binding energy of each final dicationic state was computed by single configuration RAS calculations. This calculated spectrum corresponds well to the experimental spectrum of Carroll et al. [45].

In the valence-valence Auger spectrum, in contrast to the core-valence spectra, the final singlet states are dominating and the triplet states are completely neglected in Fig. 6. This can be rationalized on the grounds that the exchange and Coulomb integrals in the intensity expression are, as confirmed by the calculations, of the same magnitude and sign, thus

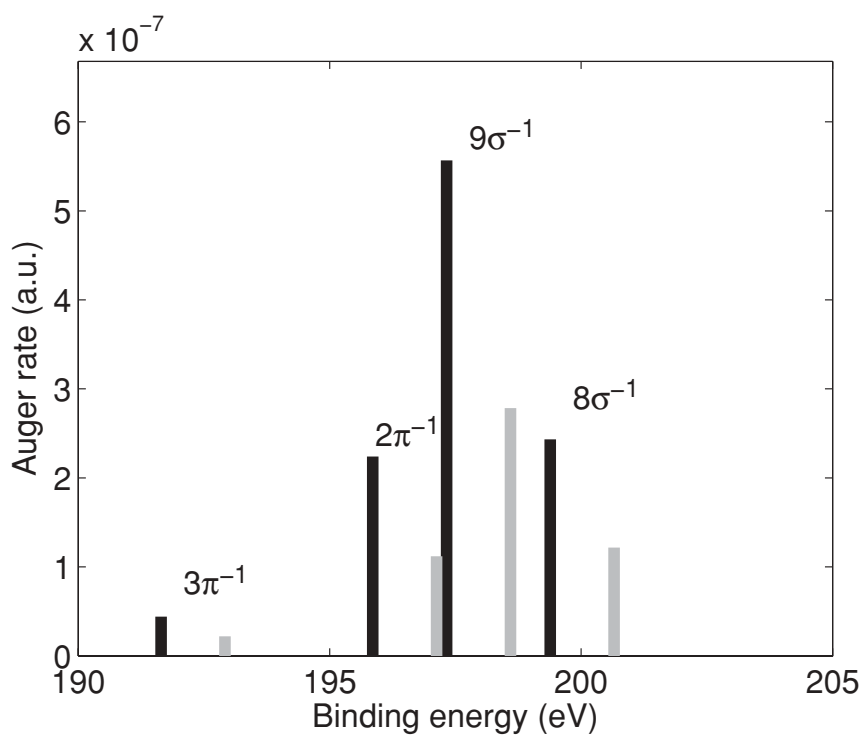

FIG. 5. The computed $\mathrm{C} 1 s^{-1} \rightarrow \mathrm{S} 2 p^{-1}(\mathrm{v})^{-1}$ core-valence Auger spectrum of the OCS molecule. The energies were obtained from MCSCF calculations and the intensities (atomic units) from the SI procedure in the independent particle, independent channel approximation. The $p_{3 / 2}$ and $p_{1 / 2}$ final states are shown in black and in gray, respectively. 


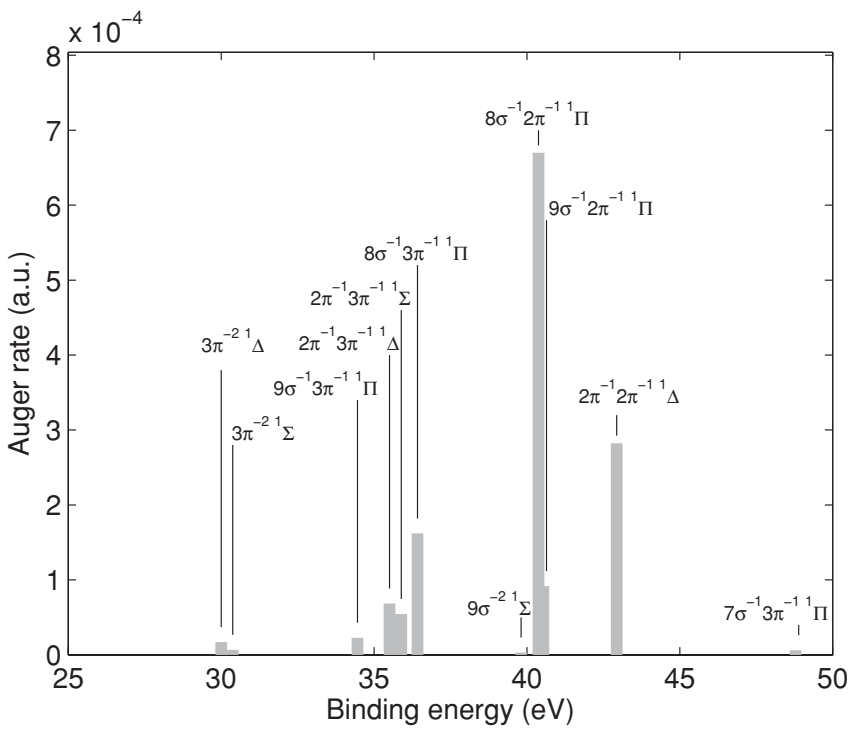

FIG. 6. The computed O $1 s^{-1} \rightarrow v_{1}^{-1} v_{2}^{-1}$ valence-valence Auger spectrum of the OCS molecule. For each final state the binding energy (eV) was obtained from MCSCF calculations and the intensity (atomic units) by the SI procedure in the independent particle, independent channel approximation. The singlet final states are assigned using bar diagrams.

counteracting on each other for the triplet amplitudes. The average intensity is about two orders of magnitude or more larger than the one observed in Figs. 4 and 5.

Finally, from an experimental point of view, we note that we would have detected, e.g., a signal for $\mathrm{C} 1 s$ to $\mathrm{S} 2 p$ crossover Auger decay if it was more than $1 / 500$ of the main Auger decay.

\section{SUMMARY}

Envisioning photoionization studies at free-electron laser sources, from where one can expect multiple core and corevalence electron spectra of many compounds, we have in the present work outlined a protocol for calculation of the corresponding electronic states. It is based on the notion of multiconfigurational self-consistent-field optimization, and the method is capable of capturing valence correlation effects and relaxation effects at the same time. This aspect is crucial for core hole states as they induce a very substantial rearrangement of the electronic cloud on their creation. Except for very small species, practical core hole MCSCF calculations though leave out dynamical correlation effects, and the correlation of the core electron itself. These two effects have been accounted for by introducing two perturbative correction schemes. We have compared the MCSCF schemes with and without dynamical correlation corrections, and also with a scheme where the valence orbital is enforced to be singly occupied in the MCSCF wave function. Moreover, we applied a recently developed open-shell spin restricted density functional theory and compared it to the results of the MCSCF schemes.

We have applied the computational approaches mentioned above to $\mathrm{O} 1 s^{-1} v^{-1}, \mathrm{C} 1 s^{-1} v^{-1}$, and $\mathrm{S} 2 p^{-1} v^{-1}$ double ionization of the OCS molecule and compared the numerical results to state-of-the-art experimental data. The DIPES spectra were measured using a highly efficient time-of-flight photoelectronphotoelectron coincidence spectroscopy technique, which provides information on the energy distribution of the dicationic states and the probability for emission of two electrons on the absorption of a single incident photon.

Generally we find that the MCSCF schemes offer a good tool to assign the experimental core-valence spectra on purely energetic grounds. The corrections applied tend to give a somewhat too large transition energy but typically within an $\mathrm{eV}$ of the experimental value. A fraction of that can probably be assigned to zero-point vibrational corrections not included in the present study. The spin-restricted DFT method was found to perform very well in comparison to the results obtained with the highly correlated $a b$ initio protocol presented in this work. This may have important ramifications for analyzing larger systems. As DFT formally correlates all electrons dynamically, no corrections were assigned. It is interesting to note that the set of functionals used give rather similar results, except one, namely LDA, which gives double ionization energies which are different by up to $5 \mathrm{eV}$ for the $\mathrm{O} 1 s$ and $\mathrm{C} 1 s$ core hole states. Such a large discrepancy is not found for the $\mathrm{S} 2 p$ core hole which can be rationalized in terms of a shortcoming of the LDA functional for deeper core electron densities.

Core-valence DIPES spectra can in general be attributed to a variety of transition channels which have different cross sections. While a complete theory for molecular double electron photoionization is outside the scope of the present work, we have provided a theory for one main channel of creation of these states, namely multiatom Auger decay, i.e., radiationless decay where one of the Auger electrons is emitted from a core orbital at a different atomic site than the photoionized core hole. This is a nonresonant analog to MARPE; like for MARPE this effect was found in the present study to be sharply dependent on the distance between the core sites involved. We find salient differences with respect to normal valence Auger. One difference is that the overall cross section is reduced by two orders of magnitude, and another difference is that the spectrum is dominated by triplet, instead of singlet, states. The latter was understood in terms of the magnitude of the Coulomb and exchange integrals in the energy expression.

\section{ACKNOWLEDGMENTS}

This work has been financially supported by the Swedish Research Council (VR), the Göran Gustafsson Foundation (UU/KTH), the Knut and Alice Wallenberg Foundation, and the Wenner-Gren Foundations, Sweden. J.N. thanks the Magnus Ehrnrooth's foundation for financial support. We are grateful to the support by the staff and colleagues at BESSY, Berlin. This work was also supported by the European Community-Research Infrastructure Action under the FP6 "Structuring the European Research Area" Programme (through the Integrated Infrastructure Initiative "Integrating Activity on Synchrotron and Free Electron Laser Science," Contract No. R II 3-CT-2004-506008). We thank Dr. Zilvinas Rinkeovicius for instructive comments on the DFT results. 
[1] J. H. D. Eland, O. Vieuxmaire, T. Kinugawa, P. Lablanquie, R. I. Hall, and F. Penent, Phys. Rev. Lett. 90, 053003 (2003).

[2] J. H. D. Eland, Chem. Phys. 294, 171 (2003).

[3] R. Feifel, J. H. D. Eland, and D. Edvardsson, J. Chem. Phys. 122, 144308 (2005).

[4] R. Feifel, J. H. D. Eland, L. Storchi, and F. Tarantelli, J. Chem. Phys. 122, 144309 (2005).

[5] E. Andersson, M. Stenrup, J. H. D. Eland, L. Hedin, M. Berglund, L. Karlsson, Å. Larson, H. Ågren, J.-E. Rubensson, and R. Feifel, Phys. Rev. A 78, 023409 (2008).

[6] E. Andersson, J. Niskanen, L. Hedin, J. H. D. Eland, P. Linusson, L. Karlsson, J.-E. Rubensson, V. Carravetta, H. Ågren, and R. Feifel, J. Chem. Phys. 133, 094305 (2010).

[7] L. S. Cederbaum, F. Tarantelli, A. Sgamellotti, and J. Schirmer, J. Chem. Phys. 85, 6513 (1986).

[8] R. Santra, N. V. Kryzhevoi, and L. S. Cederbaum, Phys. Rev. Lett. 103, 013002 (2009).

[9] M. Tashiro, M. Ehara, H. Fukuzawa, K. Ueda, C. Buth, N. V. Kryzhevoi, and L. S. Cederbaum, J. Chem. Phys. 132, 184302 (2010).

[10] H. Ågren and H. J. Aa. Jensen, Chem. Phys. 172, 45 (1993).

[11] P. Kruit and F. H. Read, J. Phys. E 16, 313 (1983).

[12] Z. Rinkevicius, I. Tunell, P. Sałek, O. Vahtras, and H. Ågren, J. Chem. Phys. 119, 34 (2003).

[13] A. W. Kay, F. J. Garcia de Abajo, S.-H. Yang, E. Arenholz, B. S. Mun, N. Mannella, Z. Hussain, M. A. Van Hove, and C. S. Fadley, Phys. Rev. B 63, 115119 (2001).

[14] V. Carravetta and H. Ågren, Chem. Phys. Lett. 354, 100 (2002).

[15] I. Cacelli, V. Carravetta, A. Rizzo, and R. Moccia, Phys. Rep. 205, 283 (1991), and references therein.

[16] V. Carravetta and H. Ågren, Phys. Rev. A 35, 1022 (1987).

[17] V. Carravetta, H. Ågren, O. Vahtras, and H. J. Aa. Jensen, J. Chem. Phys. 113, 7790 (2000).

[18] D. R. Batchelor, R. Follath, and D. Schmeisser, Nucl. Instrum. Methods A 467-468, 470 (2001).

[19] J. H. D. Eland and R. Feifel, Chem. Phys. 327, 85 (2006).

[20] R. Feifel, J. H. D. Eland, L. Storchi, and F. Tarantelli, J. Chem. Phys. 125, 194318 (2006).

[21] Z.-S. Yuan, L.-F. Zhu, X.-J. Liu, W.-B. Li, H.-D. Cheng, J.-M. Sun, and K.-Z. Xu, Phys. Rev. A 71, 064701 (2005).

[22] F. Penent, J. Palaudoux, P. Lablanquie, L. Andric, R. Feifel, and J. H. D. Eland, Phys. Rev. Letts. 95, 083002 (2005).

[23] The BESSY laboratory home page [http://www.bessy.de/].

[24] H. J. Aa. Jensen, P. Jørgensen, and H. Ågren, J. Phys. Chem. 87, 451 (1987).
[25] H. J. Aa. Jensen, H. Ågren, and J. Olsen, in Modern techniques in Computational Chemistry: MOTECC-90, edited by E. Clementi (Escom, Leiden, The Netherlands, 1990), p. 435.

[26] J. Olsen, B. O. Roos, P. Jørgensen, and H. J. Aa. Jensen, J. Chem. Phys. 89, 2185 (1988).

[27] H. J. Aa. Jensen, P. Jørgensen, H. Ågren, and J. Olsen, J. Chem. Phys. 88, 3834 (1988).

[28] C. Angeli, R. Cimiraglia, S. Evangelisti, T. Leininger, and J.-P. Malrieu, J. Chem. Phys. 114, 10252 (2001).

[29] C. Angeli, R. Cimiraglia, and J.-P. Malrieu, Chem. Phys. Lett. 350, 297 (2001).

[30] C. Angeli, R. Cimiraglia, and J.-P. Malrieu, J. Chem. Phys. 117, 9138 (2002).

[31] DALTON, a molecular electronic structure program, Release 2.0 (2005), see [http://www.daltonprogram.org], written by C. Angeli, K. L. Bak, V. Bakken, O. Christiansen, R. Cimiraglia, S. Coriani, P. Dahle, E. K. Dalskov, T. Enevoldsen, B. Fernandez, C. Hättig, K. Hald, A. Halkier, H. Heiberg, T. Helgaker, H. Hettema, H. J. Aa. Jensen, D. Jonsson, P. Jørgensen, S. Kirpekar, W. Klopper, R. Kobayashi, H. Koch, A. Ligabue, O. B. Lutnæs, K. V. Mikkelsen, P. Norman, J. Olsen, M. J. Packer, T. B. Pedersen, Z. Rinkevicius, E. Rudberg, T. A. Ruden, K. Ruud, P. Salek, A. Sanchez de Meras, T. Saue, S. P. A. Sauer, B. Schimmelpfennig, K. O. Sylvester-Hvid, P. R. Taylor, O. Vahtras, D. J. Wilson, and H. Ågren.

[32] P. Hohenberg and W. Kohn, Phys. Rev. 136, B864 (1964).

[33] W. Kohn and L. J. Sham, Phys. Rev. 140, A1133 (1965).

[34] N. D. Mermin, Phys. Rev. 137, A1441 (1964).

[35] O. Gunnarsson and B. I. Lundqvist, Phys. Rev. B 13, 4274 (1976).

[36] A. K. Theophilou, J. Phys. C 12, 5419 (1979).

[37] U. von Barth, Phys. Rev. A 20, 1693 (1979).

[38] S. M. Valone and J. F. Capitani, Phys. Rev. A 23, 2127 (1981).

[39] H. Englisch and R. English, Physica A: Stat. Theor. Phys. 121, 253 (1983).

[40] J. P. Perdew and M. Levy, Phys. Rev. B 31, 6264 (1985).

[41] D. E. Woon and T. H. Dunning Jr., J. Chem. Phys. 98, 1358 (1993).

[42] T. H. Dunning Jr., J. Chem. Phys. 90, 1007 (1989).

[43] C. J. Allan, U. Gelius, D. A. Allison, G. Johansson, H. Siegbahn, and K. Siegbahn, J. Electron Spectrosc. Relat. Phenom. 1, 131 (1972/73).

[44] G. Tu, V. Carravetta, O. Vahtras, and H. Ågren, J. Chem. Phys. 127, 174110 (2007).

[45] T. X. Carroll, D. Ji, and T. D. Thomas, J. Electron Spectrosc. Relat. Phenom. 51, 471 (1990). 